UMD-PP-97-116

hep-ph/9706268

\title{
Effective Lagrangians and Light Gravitino Phenomenology
}

\author{
Markus A. Luty円 \\ Eduardo Ponton! \\ Department of Physics \\ University of Maryland \\ College Park, Maryland 20742, USA
}

\begin{abstract}
We construct the low-energy effective lagrangian for a light gravitino coupled to the minimal supersymmetric standard model under the assumption that supersymmetry breaking is communicated to the observable sector dominantly through soft terms. Our effective lagrangian is written in terms of the spin- $\frac{1}{2}$ Goldstino (the longitudinal component of the gravitino) transforming under a non-linear realization of supersymmetry. In this lagrangian, the Goldstino is derivatively coupled and all couplings of the Goldstino to light fields are determined uniquely by the supersymmetry-breaking scale $\sqrt{F}$. This lagrangian is therefore a useful starting point for further investigation of the light gravitino in gauge-mediated supersymmetry breaking models. We show that the invisible width of the $Z$ into Goldstinos gives the constraint $\sqrt{F} \gtrsim 140 \mathrm{GeV}$.
\end{abstract}

Revised version December 1997

*mluty@physics.umd.edu

†eponton@wam.umd.edu 


\section{Introduction}

If supersymmetry plays a role in solving the gauge hierarchy problem, the scale $M_{S}$ of the masses of superpartners of observed particles cannot be much larger than $1 \mathrm{TeV}$. However, the scale $\sqrt{F}$ at which supersymmetry is spontaneously broken can be much larger [1] 国. Clearly it is important and interesting to obtain experimental information about the scale $\sqrt{F}$.

One of the few direct experimental handles on this scale comes from the fact that spontaneous breaking of supersymmetry gives rise to a massless spin- $\frac{1}{2}$ Goldstino, the Nambu-Goldstone mode associated with supersymmetry breaking. The presence of supergravity means that the Goldstino is absorbed as the longitudinal component of the spin- $\frac{3}{2}$ gravitino (superpartner of the graviton), giving rise to a spin- $\frac{3}{2}$ particle with mass [3]

$$
m_{3 / 2}=\left(2.5 \times 10^{-4} \mathrm{eV}\right)\left(\frac{F}{(1 \mathrm{TeV})^{2}}\right)
$$

In this paper, we consider models in which the gravitino mass is small compared to $M_{S}$, so that the gravitino is the lightest supersymmetric particle. This includes both gauge-mediated models [ [G, 四] and "low-scale" supergravity models [5].

We will assume that supersymmetry breaking is communicated to the observable sector via some "messenger" interactions, and that the dominant source of supersymmetry breaking in the observable sector comes through soft terms. Under this assumption, the couplings of the gravitino to matter are completely determined in terms of the supersymmetry breaking scale $\sqrt{F}$. This is in contrast to a completely model-independent approach, in which one writes the most general couplings of matter to a gravitino constrained only by non-linearly realized supersymmetry. In such a lagrangian, one finds that some of the couplings of the Goldstino are not determined by the Goldstino-independent amplitudes. The results of this paper can be interpreted as giving the values for these couplings in a large and interesting class of models. Deviations from these values would presumably occur only if supersymmetry was broken directly in the observable sector, or if "hard" breaking of supersymmetry was important. Our effective lagrangian can be used as the starting point for further phenomenological study of the gravitino in the class of models we consider.

We use our effective lagrangian to place bounds on $\sqrt{F}$. This subject has a long history going back to the earliest days of supersymmetry [6]. We find that the

\footnotetext{
${ }^{1}$ We follow conventional practice and parameterize the scale of supersymmetry breaking by a quantity $F$ that has dimension mass squared.
} 
couplings of the gravitino are suppressed by additional powers of $E / \sqrt{F}$ compared to couplings used by some authors, invalidating some of the bounds in the literature [7, 8]. An earlier version of this paper found an additional coupling of a photon to gravitino pairs of order $M^{2} E^{2} / F^{2}$, where $M \sim 50 \mathrm{GeV}$ is the coefficient of a FayetIliopoulos $D$ term; we now find that this term is not present, in agreement with Ref. 9]. We obtain a bound on $\sqrt{F}$ from the invisible width of the $Z$ to gravitinos: $\sqrt{F} \gtrsim 150 \mathrm{GeV}$. This is far from ruling out gauge-mediated or low-scale models. However, we expect similar bounds (involving unknown order-1 coefficients) in any model. Naturalness constraints tell us only that $\sqrt{F} \gtrsim 100 \mathrm{GeV}$, and we believe it is interesting that the bounds obtained in this paper begin to explore this range.

This paper is organized as follows. In Section 2, we derive the effective lagrangian using a non-linear realization of supersymmetry. In Section 3, we derive bounds on $\sqrt{F}$ from $Z$ decay. Section 4 contains our conclusions.

\section{Effective Lagrangian}

The interactions of the Goldstino at low energies are governed by low-energy theorems analogous to those which apply when global internal symmetries are spontaneously broken. The most convenient formalism for analyzing these interactions is an effective theory in which the broken symmetry (in this case supersymmetry) is realized non-linearly. This approach ensures that our results apply to any model in which supersymmetry is broken spontaneously. In this section, we review the non-linear realization of supersymmetry of Refs. 11, 12 and adapt it to the case of supersymmetry breaking in a hidden sector. (For an alternate formalism, see Ref. [13].)

Supersymmetry can be non-linearly realized on a single spinor field $\hat{\chi}$ viaf

$$
(\xi Q+\bar{\xi} \bar{Q}) \times \hat{\chi}_{\alpha}(x)=\xi_{\alpha}-i\left[\hat{\chi}(x) \sigma^{\mu} \bar{\xi}-\xi \sigma^{\mu} \overline{\hat{\chi}}(x)\right] \partial_{\mu} \hat{\chi}_{\alpha}(x) .
$$

With this definition, $\hat{\chi}$ has dimension $-\frac{1}{2}$, the same as the superspace coordinates $\theta$.

To construct supersymmetric lagrangians involving $\hat{\chi}$, we follow Ref. [12]. The field $\hat{\chi}$ can be promoted to a superfield $\Theta$ via

$$
\Theta^{\alpha}(x, \theta, \bar{\theta}) \equiv e^{\theta Q+\bar{\theta} Q} \times \hat{\chi}^{\alpha}(x)=\theta^{\alpha}+\hat{\chi}^{\alpha}(x)+\cdots,
$$

where the generators $Q$ act on $\hat{\chi}$ according to Eq. (2.1). The resulting superfield satisfies

$$
\begin{aligned}
& D_{\alpha} \Theta^{\beta}=\delta_{\alpha}{ }^{\beta}+i \bar{\Theta}^{\dot{\alpha}} \sigma_{\alpha \dot{\alpha}}^{\mu} \partial_{\mu} \Theta^{\beta}, \\
& \bar{D}_{\dot{\alpha}} \Theta^{\beta}=-i \Theta^{\alpha} \sigma_{\alpha \dot{\alpha}}^{\mu} \partial_{\mu} \Theta^{\beta} .
\end{aligned}
$$

\footnotetext{
${ }^{2}$ We use the spinor conventions of Wess and Bagger [14].
} 
We can use the superfield $\Theta$ to write manifestly supersymmetric interactions for the field $\hat{\chi}$. The most general effective lagrangian for $\hat{\chi}$ has the form

$$
\mathcal{L}=\int d^{2} \theta d^{2} \bar{\theta} \mathcal{F}\left(\Theta, \bar{\Theta}, D_{\alpha} \Theta, \ldots\right)
$$

The kinetic term for $\hat{\chi}$ is contained in the term

$$
\mathcal{L}_{\mathrm{AV}}=-\frac{F^{2}}{2} \int d^{2} \theta d^{2} \bar{\theta} \Theta^{2} \bar{\Theta}^{2}=-2 F^{2} \hat{\chi} \sigma^{\mu} \partial_{\mu} \overline{\hat{\chi}}+\cdots
$$

which is the Volkov-Akulov lagrangian. The constant $F$ has dimension 2, and parameterizes the scale of supersymmetry breaking. The canonically normalized Goldstino field is given by

$$
\chi \equiv \sqrt{2} F \hat{\chi}
$$

The lagrangian also contains higher order terms such as

$$
\delta \mathcal{L}=c F \int d^{2} \theta d^{2} \bar{\theta} D^{2} \bar{\Theta}^{2} \bar{D}^{2} \Theta^{2} \sim \frac{c}{F^{3}}\left|\partial_{\mu} \chi \sigma^{\mu \nu} \partial_{\nu} \bar{\chi}\right|^{2}+\cdots
$$

where $c$ is a dimensionless coupling constant. This gives rise to a systematic lowenergy expansion for Goldstino interactions in powers of the Goldstino energy. The parameter $c$ is somewhat model-dependent. For example, if the dynamics that breaks supersymmetry is strongly coupled with no small parameters, the effective lagrangian has the form [15]

$$
\mathcal{L} \sim F^{2} \sum_{p, \ldots, r} \int d^{2} \theta d^{2} \bar{\theta}\left(\frac{D_{\alpha}}{\Lambda^{1 / 2}}\right)^{p}\left(\frac{\bar{D}_{\dot{\alpha}}}{\Lambda^{1 / 2}}\right)^{\bar{p}}\left(\frac{\partial_{\mu}}{\Lambda}\right)^{q}\left(\Lambda^{1 / 2} \bar{\Theta}\right)^{\bar{r}}\left(\Lambda^{1 / 2} \Theta\right)^{r}
$$

where $\Lambda \sim \sqrt{4 \pi F}$ is the scale of the strong resonances. In such a model, $c \sim 1 /(4 \pi)$.

We now consider the coupling of the Goldstino to matter in models where $\sqrt{F} \gg$ $M_{S}$ and supersymmetry is communicated to the observable fields by some "messenger" sector. We assume that all fields in the messenger sector are heavy compared to $M_{S}$, and so the only light degrees of freedom are the fields of the supersymmetric standard model and the Goldstino. We further assume that the supersymmetry breaking in the observable sector occurs dominantly through soft supersymmetry breaking terms. This is the case in both gauge-mediated and gravity-mediated supersymmetry breaking (including "no-scale" models). These terms can be written in terms of supersymmetry-breaking "spurion" fields such as $\theta \theta \bar{\theta} \bar{\theta}$ [16. In order to make

\footnotetext{
${ }^{3}$ The term $\int d^{2} \theta d^{2} \bar{\theta}\left(\Theta^{2}+\bar{\Theta}^{2}\right)$ is a total spacetime derivative.
} 
these terms supersymmetric, we simply write the spurions in terms of the superfields $\Theta$ in place of the superspace coordinates $\theta$. The most general soft breaking terms can then be written as follows:

$$
\mathcal{L}_{\text {soft }}=\int d^{2} \theta d^{2} \bar{\theta} \Theta^{2} \bar{\Theta}^{2} \mathcal{O}_{\text {soft }},
$$

where

$$
\begin{aligned}
\mathcal{O}_{\text {soft }}= & -\left(m^{2}\right)_{b}^{a}\left(\bar{\Phi} e^{2 g V_{A} T_{A}} \Phi\right)_{a}{ }^{b}-\frac{1}{2}\left[B_{a b} \Phi^{a} \Phi^{b}+\text { h.c. }\right] \\
& +\frac{1}{2} \tilde{M}_{A}\left(W_{A}^{\alpha} W_{\alpha A}+\text { h.c. }\right)+\frac{1}{6}\left[A_{a b c} \Phi^{a} \Phi^{b} \Phi^{c}+\text { h.c. }\right] \\
& +\frac{1}{4}\left[g_{1} M_{D}^{2} D^{\alpha} W_{\alpha 1}+\text { h.c. }\right] .
\end{aligned}
$$

The last term is an induced Fayet-Iliopoulos term (see Eq. (2.16) below) that can appear when there is a $U(1)$ factor in the gauge group. This term should be counted as a soft supersymmetry breaking term, since it does not lead to new quadratic divergences. Although it is possible that symmetries forbid such a term, contributions of this type are induced in general models after supersymmetry breaking, as we discuss below.

We can find the component field expression of the terms above by projection with the help of Eqs. (2.3). We are interested only in terms containing at most two Goldstino fields, and we obtain

$$
\begin{aligned}
\delta \mathcal{L}_{\text {soft }}= & \left.\mathcal{O}_{\text {soft }}\right|_{0}-\left(\left.\hat{\chi}^{\alpha} D_{\alpha} \mathcal{O}_{\text {soft }}\right|_{0}+\text { h.c. }\right) \\
& -\frac{1}{4}\left(\left.\hat{\chi}^{2} D^{2} \mathcal{O}_{\text {soft }}\right|_{0}+\text { h.c. }\right) \\
& +\left.\frac{1}{2} \hat{\chi}^{\alpha} \overline{\hat{\chi}}^{\dot{\alpha}}\left[D_{\alpha}, \bar{D}_{\dot{\alpha}}\right] \mathcal{O}_{\text {soft }}\right|_{0}+O\left(\hat{\chi}^{3}\right),
\end{aligned}
$$

where $\left.\right|_{0}$ denotes the $\theta=\bar{\theta}=0$ component. In writing this result, we have omitted terms that vanish by the lowest-order $\hat{\chi}$ equations of motion.f

It is straightforward to compute the component form of Eq. (2.11). The scalar

\footnotetext{
${ }^{4}$ Some soft terms are conventionally written as $d^{2} \theta$ integrals. The $\Theta$ superfield is not chiral, so we must write these terms as full superspace integrals to make them supersymmetric.

${ }^{5}$ More precisely, these terms can be eliminated by a field redefinition to the order we are working.
} 
masses of Eq. (2.10) give

$$
\begin{aligned}
\delta \mathcal{L}_{\text {soft }}=-\left(m^{2}\right)^{a}{ }_{b}\left[\bar{\phi}_{a} \phi^{b}-\sqrt{2}\left(\bar{\phi}_{a} \hat{\chi} \psi^{b}+\overline{\hat{\chi}} \bar{\psi}_{a} \phi^{b}\right)\right. & \\
& +\left(\hat{\chi}^{2} \bar{\phi}_{a} F^{b}+\overline{\hat{\chi}}^{2} \bar{F}_{a} \phi^{b}\right)+i \hat{\chi} \sigma^{\mu} \overline{\hat{\chi}}\left(\bar{\phi} \mathcal{D}_{\mu} \phi-\mathcal{D}_{\mu} \bar{\phi} \phi\right)_{a}{ }^{b} \\
& \left.+2\left(\overline{\hat{\chi}}_{\bar{\psi}}\right)\left(\hat{\chi} \psi^{b}\right)\right]+O\left(\hat{\chi}^{3}\right) .
\end{aligned}
$$

The " $B$ term" scalar masses give

$$
\begin{aligned}
\delta \mathcal{L}_{\mathrm{soft}}=-\frac{1}{2} B_{a b}\left[\phi^{a} \phi^{b}\right. & -\sqrt{2}\left(\hat{\chi} \psi^{a} \phi^{b}+\hat{\chi} \phi^{a} \psi^{b}\right) \\
& \left.-\hat{\chi}^{2}\left(\psi^{a} \psi^{b}-F^{a} \phi^{b}-\phi^{a} F^{b}\right)\right]+ \text { h.c. }+O\left(\hat{\chi}^{3}\right) .
\end{aligned}
$$

The gaugino mass terms give

$$
\begin{aligned}
\delta \mathcal{L}_{\mathrm{soft}}=\frac{1}{2} \tilde{M}_{A}[ & -\lambda_{A} \lambda_{A}+2 \hat{\chi} \sigma^{\mu \nu} \lambda_{A} F_{\mu \nu A}+2 i \hat{\chi} \lambda_{A} D_{A} \\
& \left.-\frac{1}{2} \hat{\chi}^{2}\left(F_{A}^{\mu \nu} F_{\mu \nu A}+i F_{A}^{\mu \nu} \tilde{F}_{\mu \nu A}-2 D_{A} D_{A}+4 i \lambda_{A} \sigma^{\mu}\left(\mathcal{D}_{\mu} \bar{\lambda}\right)_{A}\right)\right] \\
& + \text { h.c. }+O\left(\hat{\chi}^{3}\right),
\end{aligned}
$$

where $\tilde{F}^{\mu \nu}=\frac{1}{2} \epsilon^{\mu \nu \lambda \rho} F_{\lambda \rho}$. The trilinear terms give

$$
\begin{aligned}
\delta \mathcal{L}_{\mathrm{soft}}=\frac{1}{6} A_{a b c}\left[\phi^{a} \phi^{b} \phi^{c}-\right. & \sqrt{2}\left(\hat{\chi} \psi^{a} \phi^{b} \phi^{c}+\phi^{a} \hat{\chi} \psi^{b} \phi^{c}+\phi^{a} \phi^{b} \hat{\chi} \psi^{c}\right) \\
-\hat{\chi}^{2}\left(\phi^{a} \psi^{b} \psi^{c}+\psi^{a} \phi^{b} \psi^{c}+\psi^{a} \psi^{b} \phi^{c}\right. & \\
& \left.\left.-F^{a} \phi^{b} \phi^{c}-\phi^{a} F^{b} \phi^{c}-\phi^{a} \phi^{b} F^{c}\right)\right]+ \text { h.c. }+O\left(\hat{\chi}^{3}\right) .
\end{aligned}
$$

Finally, the induced Fayet-Iliopoulos term gives

$$
\delta \mathcal{L}_{\text {soft }}=g M_{D}^{2}\left[D-\left(\partial^{\mu} \hat{\chi} \sigma^{\nu} \overline{\hat{\chi}}+\text { h.c. }\right) F_{\mu \nu}\right]+O\left(\chi^{3}\right) .
$$

In the lagrangian of Eqs. 2.11) through (2.16), the Goldstino field $\hat{\chi}$ is not derivatively coupled. This form of the lagrangian is useful in the energy regime $M_{\mathrm{S}} \ll E \ll F$, where $M_{\mathrm{S}}$ is the scale of the soft supersymmetry breaking masses. In this regime, Eq. (2.11) shows that the couplings of the Goldstino to observable matter fields are suppressed by positive powers of $M_{\mathrm{S}}$. 
If one is interested in the phenomenology of the Goldstino in the energy regime $E \sim M_{S}$ (or $E \ll M_{S}$ ), it is convenient to perform a field redefinition to obtain a lagrangian in which the Goldstino field is derivatively coupled. This makes manifest the fact that the couplings of the Goldstino are suppressed by positive powers of $E$ for $E \ll M_{S}$. We make this field redefinition by making a local supersymmetry transformation on the matter fields with parameter $-\hat{\chi}(x)$. In Wess-Zumino gauge, the supersymmetry transformations on component fields are generated by the operators $Q_{\mathrm{WZ}}$ defined by 14

$$
\begin{aligned}
& \left(\xi Q_{\mathrm{WZ}}+\bar{\xi} \bar{Q}_{\mathrm{WZ}}\right) \times \phi^{a}=\sqrt{2} \xi \psi^{a}, \\
& \left(\xi Q_{\mathrm{WZ}}+\bar{\xi} \bar{Q}_{\mathrm{WZ}}\right) \times \psi^{a}=i \sqrt{2} \sigma^{\mu} \bar{\xi}\left(\mathcal{D}_{\mu} \phi\right)^{a}+\sqrt{2} \xi F^{a}, \\
& \left(\xi Q_{\mathrm{WZ}}+\bar{\xi} \bar{Q}_{\mathrm{WZ}}\right) \times F^{a}=i \sqrt{2} \bar{\xi}^{\mu}\left(\mathcal{D}_{\mu} \psi\right)^{a}+2 i g \bar{\xi}(\bar{\lambda} \phi)^{a}, \\
& \left(\xi Q_{\mathrm{WZ}}+\bar{\xi} \bar{Q}_{\mathrm{WZ}}\right) \times A_{\mu A}=-i \bar{\lambda}_{A} \bar{\sigma}_{\mu} \xi+i \bar{\xi} \bar{\sigma}_{\mu} \lambda_{A}, \\
& \left(\xi Q_{\mathrm{WZ}}+\bar{\xi} \bar{Q}_{\mathrm{WZ}}\right) \times \lambda_{A}=\sigma^{\mu \nu} \xi F_{\mu \nu A}+i \xi D_{A}, \\
& \left(\xi Q_{\mathrm{WZ}}+\bar{\xi} \bar{Q}_{\mathrm{WZ}}\right) \times D_{A}=-\xi \sigma^{\mu}\left(\mathcal{D}_{\mu} \bar{\lambda}\right)_{A}-\left(\mathcal{D}_{\mu} \lambda\right)_{A} \sigma^{\mu} \bar{\xi} .
\end{aligned}
$$

We then define new fields by the field redefinition

$$
\begin{aligned}
\Phi^{a} & =e^{\xi Q_{\mathrm{WZ}}+\bar{\xi} \bar{Q}_{\mathrm{WZ}}} \times\left.\Phi^{\prime a}\right|_{\xi=\hat{\chi}}, \\
V_{A} & =e^{\xi Q_{\mathrm{WZ}}+\bar{\xi} \bar{Q}_{\mathrm{WZ}}} \times\left. V_{A}^{\prime}\right|_{\xi=\hat{\chi}}, \\
\hat{\chi} & =\hat{\chi}^{\prime} .
\end{aligned}
$$

When the lagrangian is written in terms of the primed fields defined above, the Goldstino is derivatively coupled. To establish this to all orders in $\hat{\chi}$, we will show that when $\hat{\chi}$ is taken to be a constant, the lagrangian is independent of $\hat{\chi}$. The crucial observation is that the transformation between the primed and unprimed field is "almost" a supersymmetry transformation. If $\hat{\chi}$ were a constant, and we combined the change of variables above with a transformation Eq. (2.1) acting on $\hat{\chi}$ with parameter $\xi=\hat{\chi}$, the result would be a (nonlinear) supersymmetry transformation under which the lagrangian is fully invariant. Therefore, for constant $\hat{\chi}$, the change of variables Eq. (2.18) is equivalent to

$$
\begin{aligned}
\Phi^{a} & =\Phi^{\prime a}, \quad V_{A}=V_{A}^{\prime}, \\
\hat{\chi} & =e^{-(\xi Q+\bar{\xi} \bar{Q})} \times\left.\hat{\chi}^{\prime}\right|_{\xi=\hat{\chi}} \equiv 0 \quad(\text { for } \hat{\chi}=\text { constant }) .
\end{aligned}
$$


The fact that the $\hat{\chi}$ fields are set to zero by this transformation follows directly from Eq. (2.1), and shows that the lagrangian is independent of $\hat{\chi}$ for constant $\hat{\chi}$.

When we compute the lagrangian in terms of the fields defined in Eq. (2.18), we find that there are still Goldstino couplings proportional to the gaugino mass $M$. It is convenient to eliminate these by a further field redefinition

$$
\lambda_{A}^{\prime}=\lambda_{A}^{\prime \prime}-\frac{i}{2}\left[\left(\hat{\chi} \sigma^{\mu} \bar{\lambda}_{A}^{\prime \prime}\right) \partial_{\mu} \hat{\chi}+\left(\hat{\chi} \partial_{\mu} \hat{\chi}\right)\left(\bar{\lambda}_{A}^{\prime \prime} \bar{\sigma}^{\mu}\right)\right] .
$$

When the matter field kinetic terms are written in terms of the primed fields defined in Eqs. (2.18) and (2.20), we obtain derivative couplings of the Goldstino to the matter fields with no couplings proportional to soft supersymmetry-breaking masses. (The superpotential terms contain no derivatives, and therefore do not induce Goldstino couplings.) We will assume that these kinetic terms are canonical, as is appropriate for gauge-mediated supersymmetry breaking. The final lagrangian can then be written

$$
\mathcal{L}_{\text {eff }}=\sum_{n} \frac{1}{F^{n}}\left[\mathcal{L}_{\text {matter }}^{(n)}+\mathcal{L}_{\text {gauge }}^{(n)}+\mathcal{L}_{\text {soft }}^{(n)}\right]
$$

where $n$ counts the number of powers of $\hat{\chi}$. Expressing the results in terms of the canonically normalized field $\chi$ defined in Eq. (2.6) and dropping the primes, the matter coupling terms give the usual supersymmetric terms

$$
\begin{aligned}
\mathcal{L}_{\text {matter }}^{(0)}=- & \left(\mathcal{D}^{\mu} \phi\right)^{a}\left(\mathcal{D}_{\mu} \bar{\phi}\right)_{a}-i \psi^{a} \sigma^{\mu}\left(\mathcal{D}_{\mu} \bar{\psi}\right)_{a}+F^{a} \bar{F}_{a} \\
& +i \sqrt{2} g_{A}\left[\psi^{a}\left(\lambda_{A} \bar{\phi}\right)_{a}-\phi^{a}\left(\bar{\lambda}{ }_{A} \bar{\psi}\right)_{a}\right]-\frac{g_{A}^{2}}{2}\left(\bar{\phi} T_{A} \phi\right)^{2} \\
& +\left[\frac{\partial W(\phi)}{\partial \phi^{a}} F^{a}+\frac{\partial^{2} W(\phi)}{\partial \phi^{a} \partial \phi^{b}}\left(\phi^{a} F^{b}-\frac{1}{2} \psi^{a} \psi^{b}\right)\right. \\
& \left.+\frac{1}{2} \frac{\partial^{3} W(\phi)}{\partial \phi^{a} \partial \phi^{b} \partial \phi^{c}}\left(F^{a} \phi^{b} \phi^{c}-\phi^{a} \psi^{b} \psi^{c}\right)+\text { h.c. }\right]
\end{aligned}
$$

where $W$ is the superpotential of the matter fields. (We integrate out the auxiliary fields for the gauge multiplet but not the auxiliary matter fields.) The Goldstinodependent terms are

$$
\begin{aligned}
\mathcal{L}_{\text {matter }}^{(1)}= & -2 \partial^{\mu} \chi \psi^{a}\left(\mathcal{D}_{\mu} \bar{\phi}\right)_{a}+\text { h.c. } \\
\mathcal{L}_{\text {matter }}^{(2)}=- & i \partial^{\mu} \chi \sigma^{\nu} \bar{\chi}\left(\mathcal{D}_{\mu} \bar{\phi}\right)_{a}\left(\mathcal{D}_{\nu} \phi\right)^{a}-\partial^{\mu} \chi \chi F^{a}\left(\mathcal{D}_{\mu} \bar{\phi}\right)_{a}-\partial_{\mu} \chi \psi^{a} \mathcal{D}^{\mu}(\bar{\chi} \bar{\psi})_{a} \\
& \quad-\frac{g}{2 \sqrt{2}}\left[\partial_{\mu} \chi \chi \cdot \psi^{a} \sigma^{\mu}(\bar{\lambda} \bar{\phi})_{a}-\partial_{\mu} \chi \psi^{a} \cdot \chi \sigma^{\mu}(\bar{\lambda} \bar{\phi})_{a}\right]+\text { h.c. }
\end{aligned}
$$

\footnotetext{
${ }^{6}$ It is straightforward to generalize our results to an arbitrary Kähler potential.
} 
The gauge kinetic terms give

$$
\begin{aligned}
\mathcal{L}_{\text {gauge }}^{(0)}= & -\frac{1}{4} F_{A}^{\mu \nu} F_{\mu \nu A}-i \bar{\lambda}_{A} \bar{\sigma}^{\mu}\left(\mathcal{D}_{\mu} \lambda\right)_{A} \\
\mathcal{L}_{\text {gauge }}^{(1)}= & -i \sqrt{2} \partial^{\mu} \chi \sigma^{\nu} \bar{\lambda}_{A} F_{\mu \nu A}+\text { h.c. } \\
\mathcal{L}_{\text {gauge }}^{(2)}= & \frac{i}{2} \partial_{\mu} \bar{\chi} \bar{\sigma}^{\nu} \chi \cdot F_{A}^{\mu \lambda}\left(F_{\lambda \nu A}+i \tilde{F}_{\lambda \nu A}\right)-\frac{g_{A}}{2} \partial^{\mu} \bar{\chi} \bar{\sigma}^{\nu} \chi \cdot\left(\bar{\phi} T_{A} \phi\right) F_{\mu \nu A} \\
& \quad-\frac{1}{4} \partial_{\mu} \chi\left(\mathcal{D}_{\nu} \lambda\right)_{A} \cdot \bar{\chi} \bar{\sigma}^{\nu} \sigma^{\mu} \bar{\lambda}_{A}-\frac{1}{4} \partial_{\mu} \chi \sigma^{\nu} \bar{\chi} \cdot\left(\mathcal{D}_{\nu} \lambda\right)_{A} \sigma^{\mu} \bar{\lambda}_{A} \\
& +\frac{1}{4} \partial_{\mu} \chi \partial_{\nu} \chi \cdot \bar{\lambda}_{A} \bar{\sigma}^{\mu} \sigma^{\nu} \bar{\lambda}_{A}+\frac{1}{4} \partial_{\mu} \chi \sigma^{\nu} \bar{\lambda}_{A} \cdot \partial_{\nu} \chi \sigma^{\mu} \bar{\lambda}_{A}+\text { h.c. }
\end{aligned}
$$

The soft terms give]

$$
\begin{aligned}
\mathcal{L}_{\text {soft }}^{(0)}=- & \left(m^{2}\right)^{a}{ }_{b} \bar{\phi}_{a} \phi^{b}-g_{1}^{2} M_{D}^{2} \bar{\phi} T_{1} \phi \\
& +\left[\frac{1}{2} B_{a b} \phi^{a} \phi^{b}-\frac{1}{2} \tilde{M}_{A} \lambda_{A} \lambda_{A}+\frac{1}{6} A_{a b c} \phi^{a} \phi^{b} \phi^{c}+\text { h.c. }\right], \\
\mathcal{L}_{\text {soft }}^{(2)}= & 0 .
\end{aligned}
$$

Here, $T_{1}$ is the charge of the $U(1)$ factor (see Eq. (2.10)). We have made extensive use of the $\chi$ equations of motion to simplify these expressions. The terms linear in $\chi$ are the well-known linear couplings of the Goldstino to the supercurrent [6].

The fact that the Goldstino couples only through derivatives is an automatic consequence of the non-linear representation of supersymmetry we are using. The couplings above have also been computed by various authors by taking the low-energy limit of models with linearly realized supersymmetry [7, 10]. In these calculations, the derivative couplings of the Goldstino arise from cancellations between different terms. Some results in the literature missed some of these cancellations, and obtained results in which the Goldstino is not derivatively coupled. (We are in agreement with the energy dependencies obtained in Ref. [10].) We hope that the derivation presented here is sufficiently simple and compelling to settle this issue.

\section{$3 \quad Z$ Decays into Goldstinos}

As a simple application of our formalism, we compute the decay width of the $Z$ into Goldstino pairs. There are several potential contributions from our effective

\footnotetext{
${ }^{7}$ In an earlier version of this paper, we found a contribution $\mathcal{L}_{\text {soft }}^{(2)} \propto \partial^{\mu} \chi \sigma^{\nu} \bar{\chi} F_{\mu \nu}+$ h.c. This term is canceled by a similar term from the $D_{1}$ equation of motion, giving a result in agreement with Ref. [9].
} 
lagrangian. The term $\partial^{\mu} \chi \chi F \mathcal{D}_{\mu} \bar{\phi}$ in Eq. (2.24) gives rise to a coupling of the $Z$ to Goldstino pairs from terms where $F$ and $\phi$ have vacuum expectation values. However, in the minimal supersymmetric standard model, $\langle F\rangle$ comes only from the $H_{1} H_{2}$ term in the superpotential, and this contribution to the $Z$ coupling vanishes identically. The term proportional to $D_{A}$ in Eq. (2.27) gives a direct coupling of Goldstino pairs to the $Z$ via

$$
\delta \mathcal{L}=\frac{1}{2 F^{2}} \partial^{\mu} \bar{\chi} \bar{\sigma}^{\nu} \chi\left\langle D_{Y}\right\rangle B_{\mu \nu}+\text { h.c. },
$$

where

$$
\left\langle D_{Y}\right\rangle=\frac{e v^{2}}{2} \frac{\cos 2 \beta}{\sin 2 \theta_{W}} \simeq(75 \mathrm{GeV})^{2}\left(\frac{\cos 2 \beta}{0.5}\right) .
$$

This is small for $\tan \beta$ near 1 , but we regard this as a fine-tuned limit. This gives a contribution to the invisible width of the $Z$

$$
\Gamma(Z \rightarrow \chi \chi)=\frac{\left\langle D_{Y}\right\rangle^{2} M_{Z}^{5}}{96 \pi F^{4}} .
$$

We place a limit on $F$ by demanding that this not spoil the agreement between the invisible $Z$ width as calculated in the standard model and measured at LEP. We therefore impose $\Gamma(Z \rightarrow \chi \chi)<7.5 \mathrm{MeV}$, which is 3 times the experimental uncertainty from the combined LEP average (the theoretical uncertainty is negligible) [18]. This gives a bound

$$
\sqrt{F}>(140 \mathrm{GeV})\left(\frac{\cos 2 \beta}{0.5}\right)^{1 / 4} .
$$

\section{Conclusions}

We have computed the low-energy couplings of the longitudinal components of a light gravitino (the Goldstino) to the minimal supersymmetric standard model under the assumption that supersymmetry breaking is comminicated from a hidden sector via soft-breaking terms. We have shown that these couplings are derivatively coupled to all orders in the Goldstino field $\chi$, and we have explicitly worked out the lagrangian to second order in $\chi$. All couplings of the Goldstino are uniquely determined in terms of the scale of supersymmetry breaking $\sqrt{F}$. We used this lagrangian to obtain a weak bound on $\sqrt{F} \gtrsim 140 \mathrm{GeV}$ from the decay of the $Z$ into Goldstinos. This lagrangian can be used as the starting point for further phenomenological investigation of the Goldstino in gauge-mediated and no-scale models. 


\section{Acknowledgments}

M.A.L. would like to thank R.N. Mohapatra for discussions.

\section{References}

[1] M. Dine, W. Fischler, M. Srednicki, Nucl. Phys. B189, 575 (1981); S. Dimopoulos, S. Raby, Nucl. Phys. B192, 353 (1981); L. Alvarez-Gaumé, M. Claudson, M.B. Wise, Nucl. Phys. B207, 96 (1982).

[2] J. Polchinski, L. Susskind, Phys. Rev. D26, 3661 (1982); L. Hall, J. Lykken, S. Weinberg, Phys. Rev. D27, 2359 (1983).

[3] D.V. Volkov, V.A. Soroka, JETP Lett. 18, 312 (1973); S. Deser, B. Zumino, Phys. Rev. Lett. 38, 1433 (1977).

[4] M. Dine, A.E. Nelson, hep-ph/9303230, Phys. Rev. D48, 1277 (1993); M. Dine, A.E. Nelson, Y. Shirman, hep-ph/9408384, Phys. Rev. D51, 1362 (1995); M. Dine, A.E. Nelson, Y. Nir, Y. Shirman, hep-ph/9507378, Phys. Rev. D53, 2658 (1996).

[5] J. Ellis, K. Enqvist, D.V. Nanopoulos, Phys. Lett. 147B, 99 (1984).

[6] P. Fayet, Phys. Lett. 70B, 461 (1977); Phys. Lett. 84B, 416 (1979); Phys. Lett. 86B, 272 (1979).

[7] T. Moroi, H. Murayama, M. Yamaguchi, Phys. Lett. 303B, 289 (1993); T. Gherghetta, hep-ph/9607448, Nucl. Phys. B485, 25 (1997).

[8] J.A. Grifols, R.N. Mohapatra, A. Riotto, hep-ph/9610458.

[9] T.E. Clark, Taekoon Lee, S.T. Love, Guo-Hong Wu, hep-ph/9712353.

[10] A. Brignole, F. Feruglio, F. Zwirner, hep-ph/9703286.

[11] D.V. Volkov, V.P. Akulov, JETP Lett. 16, 438 (1972); E.A. Ivanov, A.A. Kapustnikov, Jour. Phys. A11, 2375 (1978).

[12] S. Samuel, J. Wess, Nucl. Phys. B221, 153 (1983); See also Ref. [14.

[13] T.E. Clark and S.T. Love, Phys. Rev. D54, 5723 (1996). 
[14] J. Wess, J. Bagger, Supersymmetry and Supergravity, Second Edition, (Princeton, 1992).

[15] M.A. Luty, hep-ph/9706235, to be published in Phys. Rev. D.

[16] L. Girardello, M.T. Grisaru, Nucl. Phys. B194, 65 (1982).

[17] S. Dimopoulos, M. Dine, S. Raby, S. Thomas, hep-ph/9601367, Phys. Rev. Lett. 76, 3494 (1996); S. Dimopoulos, S. Thomas, J.D. Wells, hep-ph/9604452, Phys. Rev. D54, 3283 (1996); S. Ambrosanio, G.L. Kane, G.D. Kribs, S.P. Martin, S. Mrenna, hep-ph/9605398, Phys. Rev. D54, 5395 (1996); K.S. Babu, C. Kolda, F. Wilczek, hep-ph/9605408, Phys. Rev. Lett. 77, 3070 (1996).

[18] Particle Data Group (R.M. Barnett et. al.), Phys. Rev. D54, 1 (1996). 